\title{
Overview of 3D surface digitization technologies in Europe
}

\author{
Nicola D'Apuzzo* \\ Homometrica Consulting - Dr. Nicola D'Apuzzo, Culmannstrasse 59, 8006 Zurich, Switzerland
}

\begin{abstract}
This paper presents an overview of the different 3D surface digitization technologies commercially available in the European market.

The solutions for 3D surface measurement offered by major European companies can be divided into different groups depending on various characteristics, such as, technology (e.g. laser scanning, white light projection), system construction (e.g. fix, on $\mathrm{CMM} / \mathrm{robot} / \mathrm{arm}$ ) or measurement type (e.g. surface scanning, profile scanning). Crossing between the categories is possible, however, the majority of commercial products can be divided into the following groups: (a) laser profilers mounted on CMM, (b) portable coded light projection systems, (c) desktop solutions with laser profiler or coded light projection system and multi-axes platform, (d) laser point measurement systems where both sensor and object move, (e) hand operated laser profilers, hand held laser profiler or point measurement systems,(f) dedicated systems.

This paper presents the different 3D surface digitization technologies and describes them with their advantages and disadvantages. Various examples of their use are shown for different application fields. A special interest is given to applications regarding the 3D surface measurement of the human body.
\end{abstract}

Key words: 3D surface scanning, laser scanning, laser profiler, white light projection, hand held scanner, human body

\section{INTRODUCTION}

The considerations presented in this paper are made upon analysis and surveying of the 3D digitization market in Europe. Similar remarks can be made for the north American, south American and Asian markets. However, slightly different markets could respect the different distribution of companies developing and producing measurement systems. In fact, because of historical reasons, the production and development of 3D digitization solutions based on white light projection is concentrated in Europe, especially in Germany, whereas solutions based on laser scanning are mainly produced and developed in north America.

Technologies used commercially for the measurement of the surface of objects with micro to macro sizes (i.e. from some $\mathrm{cm}$ up to several meters) can be divided fundamentally into two groups: systems based on laser scanning and systems based on white light projection. The used equipment is different, however they are based on the same principle: triangulation.

Laser scanning systems employs lasers to project a spot, a line, multiple lines, or patterns onto a surface, whereas a light sensor, usually a camera, acquires the scene. The three elements laser, light sensor and object surface form a triangle. When the geometrical disposition of the laser and the light sensor are known, the distance of the object surface to the laser scanning device can be easily determined by triangulation. To measure surface areas the laser spot, line, multiple lines or pattern have to move over the area (i.e. scan the surface). For this process, different methods can be used, e.g. mirrors systems, electro-mechanical systems, hand operated systems.

3D measurement systems based on white light employ projectors instead of laser light sources, to project light patterns onto the surface. The measurement principle remains the same: triangulation. A triangle is formed by projector, camera and object. In this case, to cover entire surface parts, surface areas are illuminated by the employed projector. Special codes are used to determine the origin of the light source, e.g. binary codes, color codes.

The two different technologies result in various surface scanning devices with different characteristics. Some examples are laser profilers mounted on CMM, portable coded light projection surface digitizer, portable laser scanners, hand held surface digitizers. An extensive description of the different solutions available in the market will be given in the next section, followed by examples of applications in various fields.

*info@homometrica.ch; phone +41-79-3939-786; fax +41-44-3623-297; www.homometrica.ch 


\section{ACTUAL STATE OF TECHNOLOGY}

The remarks presented in this section are based upon the survey of the European market of solutions for the non-contact 3D measurement of the surface of objects with micro to macro sizes, i.e. objects having dimensions from some $\mathrm{cm}$ to several meters.

\subsection{Subdivision of the market}

The solutions for 3D surface measurement offered by the major companies can be divided into different group depending on how they are categorized. The following table gives an overview of the different groups:

\begin{tabular}{|l|l|l|}
\hline Measurement type & Technology & System \\
\hline surface measurement & laser scanning & fix system \\
\hline measurement of profiles & coded light projection & system moves (CMM, robot) \\
\hline point measurement & interferometry & hand held / hand operated \\
\hline & time-of-flight & object moves (rotating platform) \\
\hline & & both system and object move \\
\hline
\end{tabular}

Crossing between all the categorizations is possible, e.g. a coded light projection surface measurement system mounted on a robot or a hand held laser scanning surface profiler. However, the majority of the products present in the European market can be divided into the following groups:

(a) laser profilers mounted on CMM,

(b) portable coded light projection systems,

(c) desktop solutions with laser profiler or coded light projection system and multi-axes platform,

(d) laser point measurement systems where both sensor and object move,

(e) hand operated laser profilers, hand held laser profiler or point measurement systems,

(f) dedicated systems (e.g. full human body scanner).

The following sections will present the different groups and give examples of products developed by European companies.

\subsection{Laser profilers mounted on CMM}

Laser profilers are extensively used for industrial measurements. They are usually composed of a camera and a laser light source included in a compact and stable case. A laser line is projected onto the object surface and its profile is digitized very accurately at a very high rate. Laser profilers have to be moved over an object to digitize its surface. In the industrial measurement field, they are usually mounted on CMM (coordinate measurement machine), allowing a very accurate and automatic digitization of complex shapes in relatively short time. Moreover, the measured 3D point cloud does not need further processing, as for example data merging or alignment. The advantages of laser profilers are the very compact size $(10-30 \mathrm{~cm})$, the extremely high precision (range of $\mu \mathrm{m})$ and the high rate data acquisition.

Variations of the typical sensor setup (one camera and one laser light source) are present in the market to fulfill any special requirement, as for example laser profilers with different basis, multiple laser lines, multiple cameras or multiple sensors. Figure 1 shows three products from European companies.
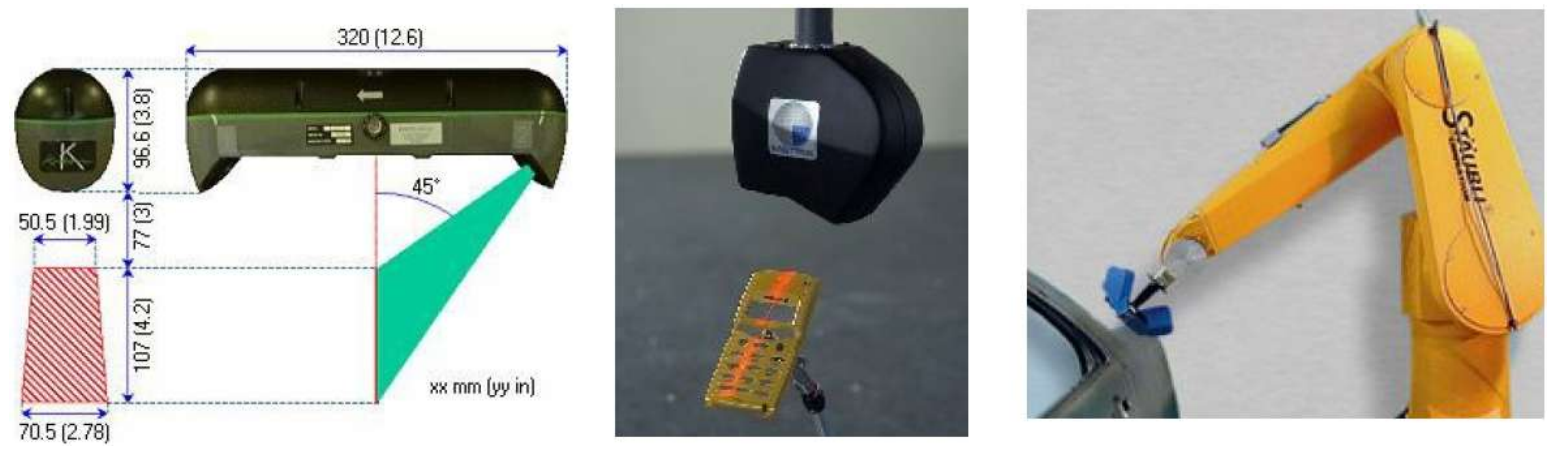

Figure 1: Examples of laser profilers from European companies. From left to right: Kreon KLS171 (France); Metris LC15 (Belgium) mounted on a CMM-machine; 3D Alliance PHT1 (Germany) mounted on a robot. 


\subsection{Portable coded light projection systems}

This represent a second group of surface measurement systems extensively used in Europe. Developed and produced from European companies, exist only coded light portable measurement systems. However, north American and Asian companies offer portable laser scanning solutions which have similar characteristics.

Portable coded light projection systems are usually composed of a single or multiple cameras and a digital white light projector. These systems are relatively compact in size and can be therefore placed and moved easily. Moreover, they can be easily adjusted to different field-of-views by changing lenses and/or displacing the projector and cameras.

The projector serves to project coded light patterns onto the object surface and the cameras serve to acquire the scene. In this way, the illuminated area is digitized very accurately in a single acquisition. The main advantage of these systems is, in fact, the fast and precise digitization of relatively large surface areas. On the other hand, the digitization of complex objects usually requires the acquisition from multiple directions. This implies additional post processing of the acquired data, as for example, merging and aligning the 3D data from the multiple acquisitions. The non-automatism of the digitization process is the main disadvantages of portable coded light projection systems.

More convenient are the systems allowing the synchronization of multiple units for an acquisition from multiple directions in shorter time. Rotating and/or moving platforms can also be used to automate the acquisition process.

An additional important feature of these systems is the simultaneous acquisition of high quality texture images (which is not the case for laser profilers). These can serve for the generation of fully textured 3D computer model of the measured object. Variations of the typical sensor setup (one or multiple cameras and one projector) are present in the market to fulfill any special requirement, as for example systems with variable basis, multiple projectors, mirrors systems or additional integrated illumination. Figure 2 shows three systems developed by European companies.
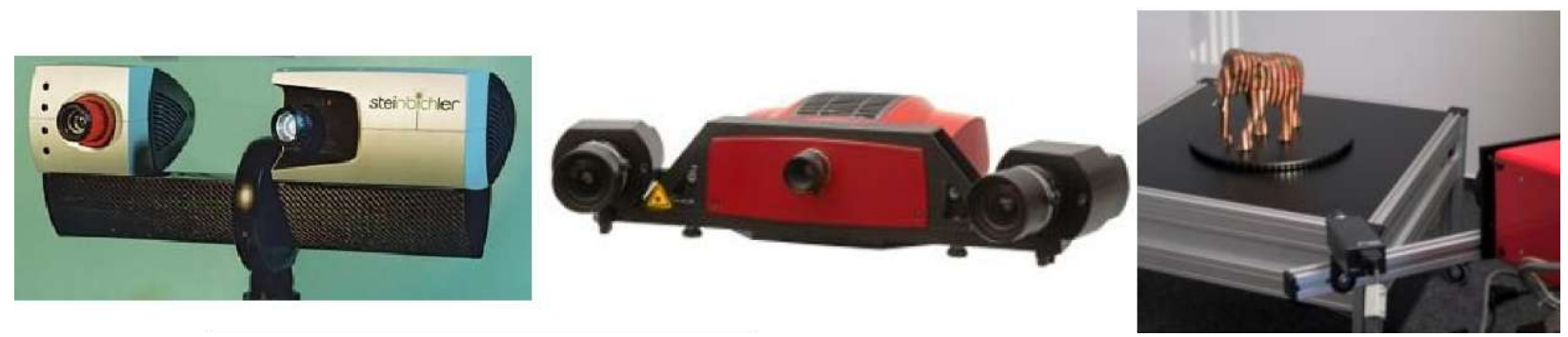

Figure 2: Examples of portable coded light projection systems from European companies. From left to right: Steinbichler COMET IV (Germany), with variable basis and additional led illumination; GOM ATOS-III (Germany), with two cameras; Polygon Technolgy QTSculptor (Germany), with rotating platform.

\subsection{Desktop solutions with laser profiler or coded light projection system and multi-axes platform}

This group of systems represents desktop solutions designed to measure small object fully automatically. The object to be measured is fixed on a support and the measurement process of the entire object is completed by moving and rotating the object and/or the sensor. Different sensor technologies can be employed, but the mostly used are coded light projection and laser profiling. By the first, surface areas are acquired by single scans and the coverage of the entire surface of the object is achieved by displacing and rotating the object. By laser profiling, a laser line is displaced over the object and profiles are acquired; in this case both the sensor and the object have to move to scan it entirely.

The main advantage of these systems is the full automatic acquisition process.

Various system setups exists, with moving platform from 2-axes ( $\mathrm{x}-$ and $\mathrm{y}$-displacement) up to 7-axes (x-,y-,zdisplacement, roll, pitch, yaw, rotation). Figure 3 shows two examples of systems developed by European companies.
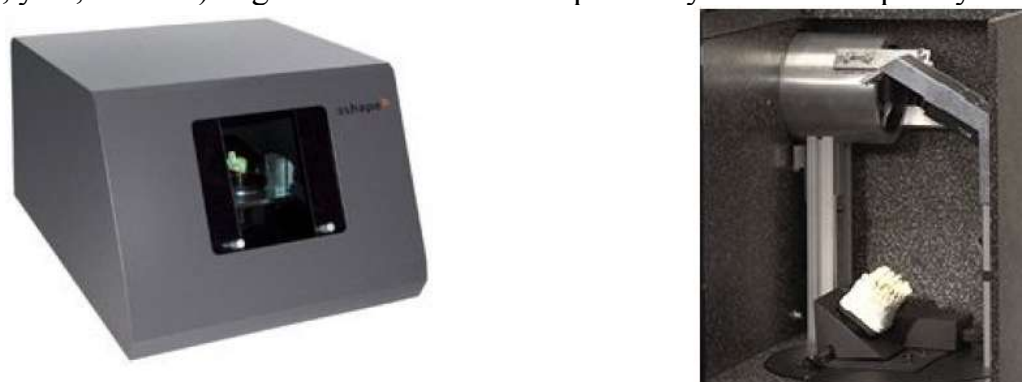

Figure 3: Examples of desktop 3D measurement systems from European companies. From left to right: 3Shape S-200 (Denmark), using laser profiling and 3-axes platform; IVB-Jena HSdig Volume (Germany), using coded light projection with mirrors system and 5 -axes platform. 


\subsection{Laser point measurement systems where both sensor and object move}

These systems can be considered as using the simplest method to acquire surfaces in 3D. They basically measure single laser spots by triangulation. The coverage of surface parts is achieved by displacing and rotating both the sensor and the object. The measurement process proceeds automatically. The main disadvantage of such systems is the long acquisition time. The main advantage is the reduced cost in comparison with other automatic measurement systems.

The variety of system setups is very large, because of the required displacement and/or rotating mechanisms of both object and sensor. Figure 4 shows two examples of systems developed by European companies.
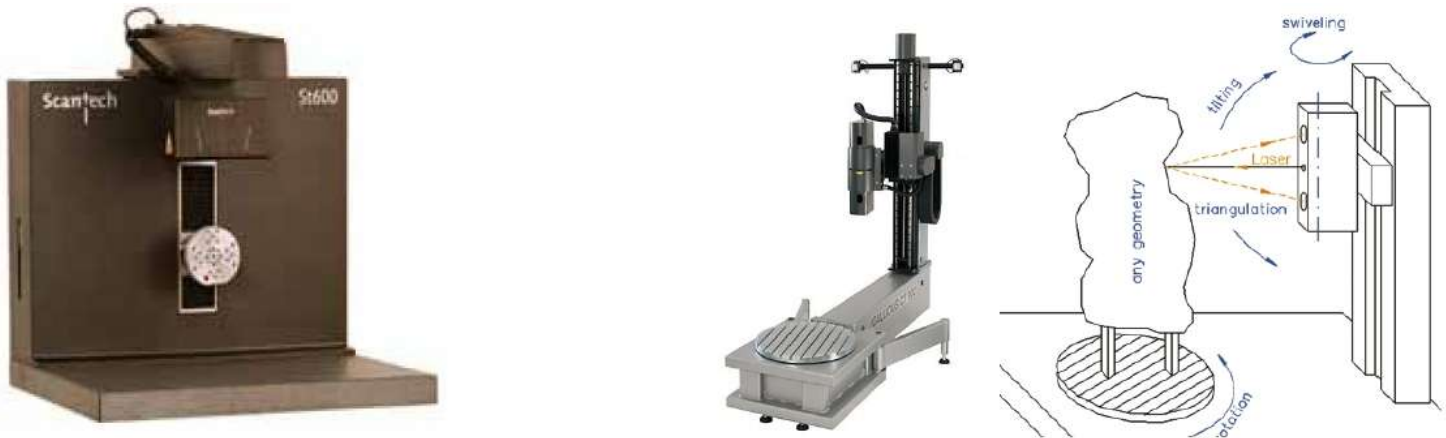

Figure 4: Examples of laser point measurement systems where both sensor and object move. From left to right: Scantech st600 (Denmark), with 2-linear-axes sensor movement and 1-linear- and 1-rotation-axis of the object; Callidus CT900 (Germany) scanner and measurement principle (1-linear-axis and 2-rotation-axes of the sensor and 1-rotation-axis of the object).

\subsection{Hand operated laser profiler, hand held laser profiler or point measurement systems}

In the case of hand operated or hand held laser profilers, the movement task is performed by hand instead of by motorized systems, i.e. the object or the sensor are displaced by hand to process the digitization of the surface. These systems exists in different forms, two are the most common: laser profiler mounted on a mechanical arm, hand held laser profiler or laser point measurement.

The main advantages of these systems are the manual control of the scanning process and the real-time view of the acquired $3 \mathrm{D}$ data. The user can in this way digitize complex objects in shorter time as the full automatic systems.

In the case of laser profiler mounted on mechanical arms, the scanning process is controlled manually by moving the laser profiler over the object. The articulated arm delivers in real time the position and orientation of the sensor very accurately, resulting in an immediate digitization of the surface scanned by the laser profiler.

Hand held laser profilers or laser point measurement systems allow a complete freedom of movement. Different methods are available to determine in real-time the position and orientation of the sensor: laser tracking, photogrammetry, electromagnetic tracking. Laser tracked systems employs (the very expensive) laser tracking technology to determine very precisely the position and orientation of the hand-held scanner marked with special targets. By electro-magnetically tracked systems, the hand-held scanner are equipped with EM sensors whose position is determined in real time by EM-tracking devices. The accuracy of such systems is lower. Photogrammetric systems uses photogrammetric technology, i.e. image information, to establish the position and orientation of the sensors relatively to the object.

Figure 5 shows three examples of systems produced by European companies.
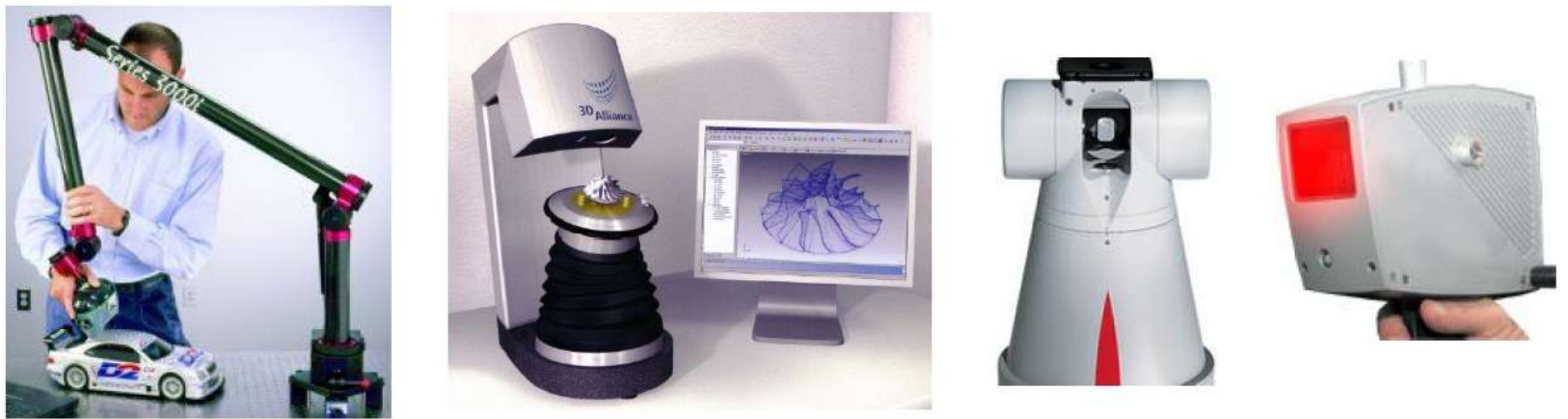

Figure 5: Examples of hand operated or hand held measurement systems from European companies. From left to right:Kreon KLS51 (France) laser profiler mounted on mechanical arm; hand operated scanner 3D Alliance Hexman (Germany), fix laser profiler with 5axes hand operated platform; laser tracker Leica $L T(D) 800$ and hand held laser point scanner T-scan (Switzerland). 


\subsection{Dedicated systems}

A large group of 3D surface measurement systems is composed of all the solutions customized for specific applications, i.e. the systems are dedicated for the acquisition of specific objects and are designed according to the specific task. Both laser scanning and coded light methods can be used, multiple sensors are usually employed and/or specific movements of the sensors are designed.

Figure 6 shows three different examples of systems dedicated to special measurement tasks.
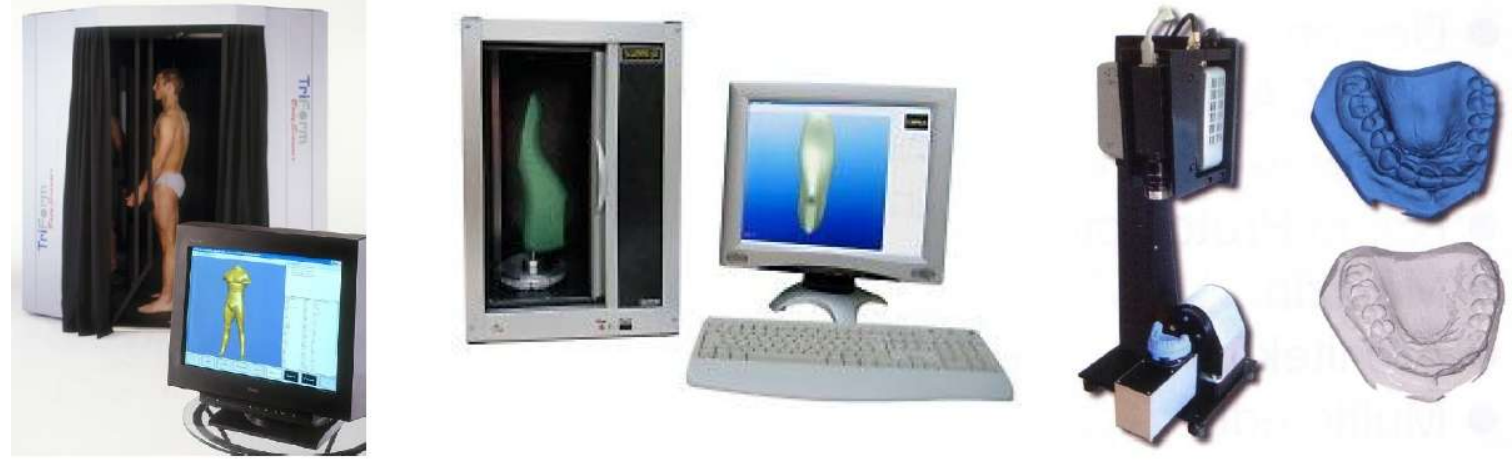

Figure 6: Examples of dedicated systems from European companies. From left to right: full body scanner Wick and Wilson TriForm3DBody Scanner (UK), with four coded light projection systems each with one projector and two cameras; scanner for foot lasts Scanny3D Base (Italy), with laser point measurement system with two cameras and 1-axis linear movement and 1-axis rotation platform; dental cast scanner RSI digiSCAN dental (Germany), with coded light measurement system composed of one camera and one projector and 3 -axes platform.

\section{EXAMPLE OF APPLICATIONS}

This section presents examples of applications of 3D surface digitization for the four major groups of users of this technology: industrial measurements, cultural heritage, consumer industry and medical sciences.

\subsection{Industrial measurements ${ }^{1}$}

A typical application of 3D surface digitization in the field of industrial measurement is the dimensional inspection of produced parts. This process is very important both in the design as well as in the production phase of a product. The inspection systems not only must be precise and accurate, but they also must be versatile enough to allow rapid measurement of parts or tools in different parts of the process with as much information as possible.

In the last decades, dimensional inspection processes have used coordinate measurement machines (CMM). Great measurement accuracy can be obtained with these machines. Nonetheless, this technology also has its inconveniences, for example: the piece to be measured must be fasten to the measuring table; as CMMs require contact with the part they are not reliable on soft materials or deformable pieces; the measuring process is slow and not massive, for which reason there is not all of the information on the areas with free surfaces; CMMs are not transportable and requires special set-up for the installations.

In the last years, more and more companies use 3D optical surface measuring systems that allow to solve these problems. As example, is shown in Figure 7 the dimensional inspection of turbine blades by using the optical measuring system Triple of the Spanish company Nub3d.

The system applied in this example, uses the coded white light projection technology. To measure the complete surface of turbine blades, multiple acquisitions are required. These are then merged by standard alignment procedures. The accuracy of the measurement achieved by this technology is about $10 \mu \mathrm{m}$.
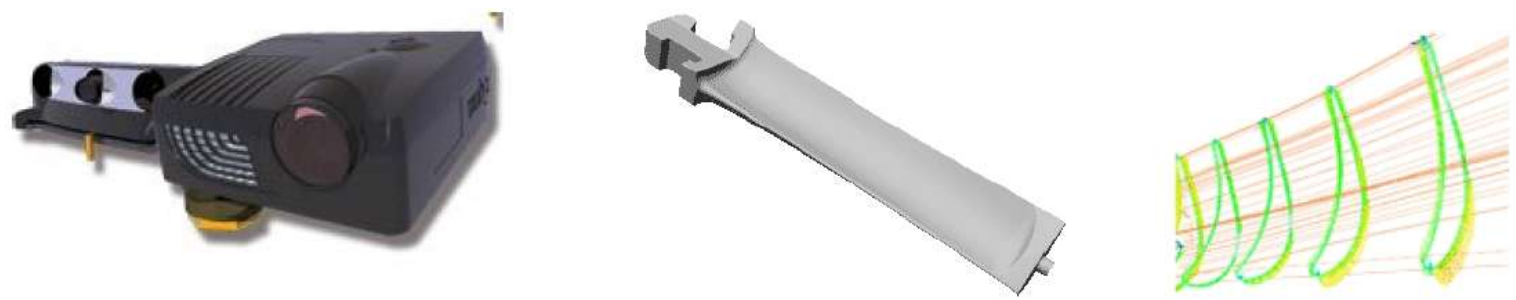

Figure 7: Optical 3D digitizer Nub3D Triple (Spain) (left); measured turbine blade (center); profile analysis (right). 


\subsection{Cultural heritage}

Cultural heritage represents an established user group of optical 3D measurement systems since the non-contact characteristic of the technology plays a relevant role. In fact, the non-contact 3D digitization of historical objects offers many advantages for their preservation. Primary, the object can be analyzed without touching it and therefore without damaging it. Secondly, a records database of ancient or historical object can be established. Third, the digitized objects can be viewed by a larger amount of people by setting up digital museums.

An interesting example is shown in Figure 8: the 3D measurement of dinosaur skeletons by laser scanning conducted at different zoological Museums in Europe by the Computer Vision and Remote Sensing Laboratory of the Berlin University of Technology, Germany.

Dinosaur skeletons are complex objects with irregular structures so that laser scanning technology will achieve best results to determine the shape of the bones. Multiple acquisitions are required to scan the entire skeleton of dinosaurs. For example, the skeleton of the Indian elephant $\left(1.70 \times 1.50 \times 0.70 \mathrm{~m}^{3}\right)$ of Zoological Museum of Copenhagen (on the right) was scanned from 7 scanner positions and with 15 reference points, resulting in over 920,000 points with an accuracy of the measurement better than $1 \mathrm{~mm}$.
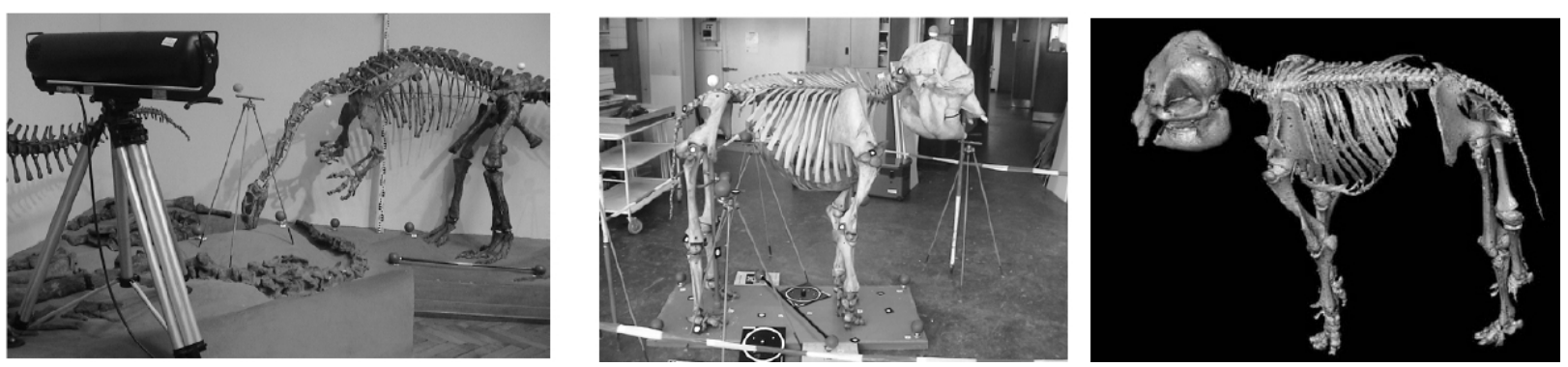

Figure 8: Left: scanning setup (Laser Scanner Mensi S25) with dinosaur skeleton and reference points. Center and right: scanning setup and measured 3D point cloud of the Juvenile Indian elephant skeleton of the zoological museum of Copenhagen (Denmark).

\subsection{Consumer industries ${ }^{3}$}

In the last years, the use of reverse engineering and rapid prototyping technologies have drastically increased for the production of consumer products. For these procedures, optical 3D surface measurement techniques are the most adequate. Typical examples of products where 3D techniques are used for inspection and reverse engineering are mobile phones, shavers, worktools, electrical connectors and other small plastic objects.

Such products are usually composed of different high precision injection moulded plastic parts with complex geometries. The attractive design, well-thought ergonomics, and basically the fitting of the different shells requires a thorough inspection. As today's pressure on manufacturing is very higher, many companies decided to invest in new modern inspection systems that enables fast and accurate scanning of full parts.

An example is given by the Atlas Copco Electric Tools, (Winnenden, Germany). The company manufactures a variety of electric tools, known as "AEG" or "Milwaukee" tools. Atlas Copco Electric Tools installed a Metris LC50 laser profiler (Belgium) on a DEA Global Image coordinate measuring machine (CMM) to verify the full 3D geometry of the housings of produced tools ${ }^{3}$.

Atlas Copco Electric Tools, manufactures a variety of electric tools such as percussion drills, screwdrivers, demolition hammers, etc. The Figure 9 shows the example of measurement and inspection of the housing of an electric drill.
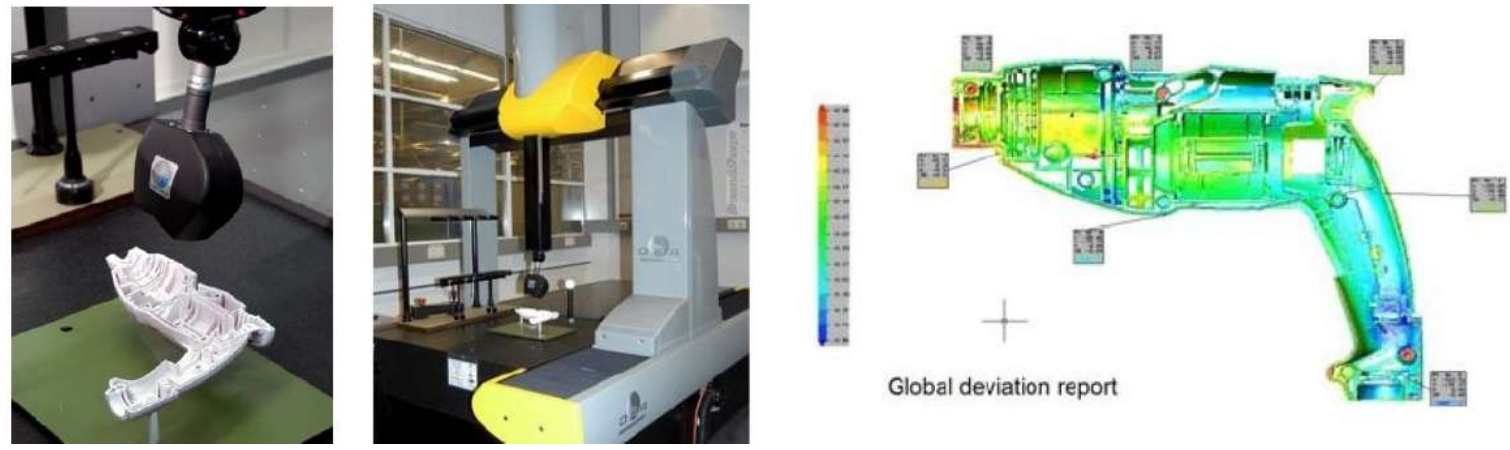

Figure 9: Metris LC50 (Belgium) laser profiler is digitizing a tool housing (left); DEA Global Image CMM with scanner (center); comparison of measured 3D point cloud of the part to the reference CAD model. 


\subsection{Medical sciences}

Medical sciences are also interested in the 3D scanning technologies because of their high accuracy, fast acquisition and non-contact characteristics. A good example of the advantages of optical 3D measurement technologies in the medical field can be found in dentistry.

The 3D measurement of dental casts brings in the orthodontics many advantages and new opportunities. Several 3D scanners specially designed for this applications exists in the market. They allow a precise, full automatic and fast 3D scanning of full dental casts, dies/stumps, inlay preparations, bridge preparations, bites/antagonists, wax-ups, and superstructures.

The acquired data can be useful for many reasons, as for example: 3D databases of dental casts accessible in a local area network reduce storage costs and give easy and instantaneous access to a patient's teeth profile;3D software solutions allow a simplified design of caps, crowns, inlays and bridges from the scanned data; patient's dentitions can be analyzed in detail, for example to assess the efficiency of an orthodontics treatment.

Figure 10 shows the example of a 3D dental scanner 3Shape D-200 (Denmark), some sample data of 3D scans, as well as an example of digital design of dental restorations.
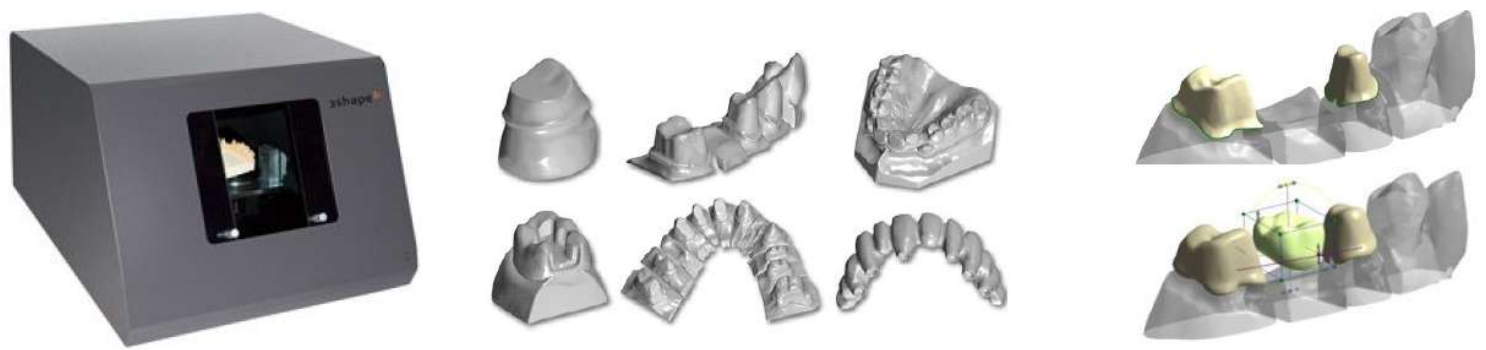

Figure 10: 3Shape D-200 Dental 3D Scanner (Denmark) (left); sample measured data (center); 3Shape DentalDesigner software examples (right).

\section{3D HUMAN BODY SURFACE MEASUREMENT ${ }^{5}$}

In the next two sections, special weight is given to the utilization of 3D digitization technologies for the 3D measurement of the human body. Since, the market in this special field is not yet sufficiently exploited, the discussion will not be limited to Europe only. In fact, even though, precedence was given to European products or application, some valuable products and some interesting applications from north America and Asia are presented.

The next sections will give a short description of the different technologies used commercially for the measurement of the human body: (a) laser scanning, (b) projection of light patterns, (c) combination modeling and image processing.

\subsection{Laser scanning}

Laser scanning technology consists of using laser light sources to project on the human body one or more thin stripes. Simultaneously, light sensors acquire the scene and by applying simple geometrical rules, the surface of the human body is measured. To assure the inoffensiveness of the light beam, only eye-safe lasers are used. Special optical systems and mirrors are used for the generation of stripes from a single laser light beam.

A laser scanner unit is composed of the laser, the optical system and the light sensor. This unit is moved across the human body to digitize the surface. The type of movement and the number of employed units can vary depending on the human body parts to be measured. For example, the full body scanner of Hamamatsu Photonics (Japan) consists of four scanner units that move vertically synchronously along four pillars (Figure $11 \mathrm{left}$ ). A second example is the head scanner of Cyberware (USA). In this case, a unique scanner unit moves in circle around the head of a person (Figure 11 center). As last example is shown the foot scanner of Vitronic (Germnay): the scanner is composed of three units, which moves horizontally, two laterally and one from the bottom (Figure 11 right).

The high costs for production of hardware components for the laser scanning technology have to be considered as disadvantage. Additionally to the laser, the light sensor and the optical system, also precise electric motors have to be used for the displacement of the scanner units. Moreover, the complete scanner system has to be calibrated so that the geometrical disposition of all the elements can be determined exactly. A second disadvantage of this method is the time required for the digitization of large surfaces. There is no problem for the measurement of extremities as feet and hands, since these body parts can be kept immobile for some seconds. But, in the case of the measurements of head or full body, it is practical impossible to stay immobile for several seconds. Uncontrolled movements as breathing or muscle contraction can generate errors, especially in the case of face measurement with its small scale and the required high resolution. 

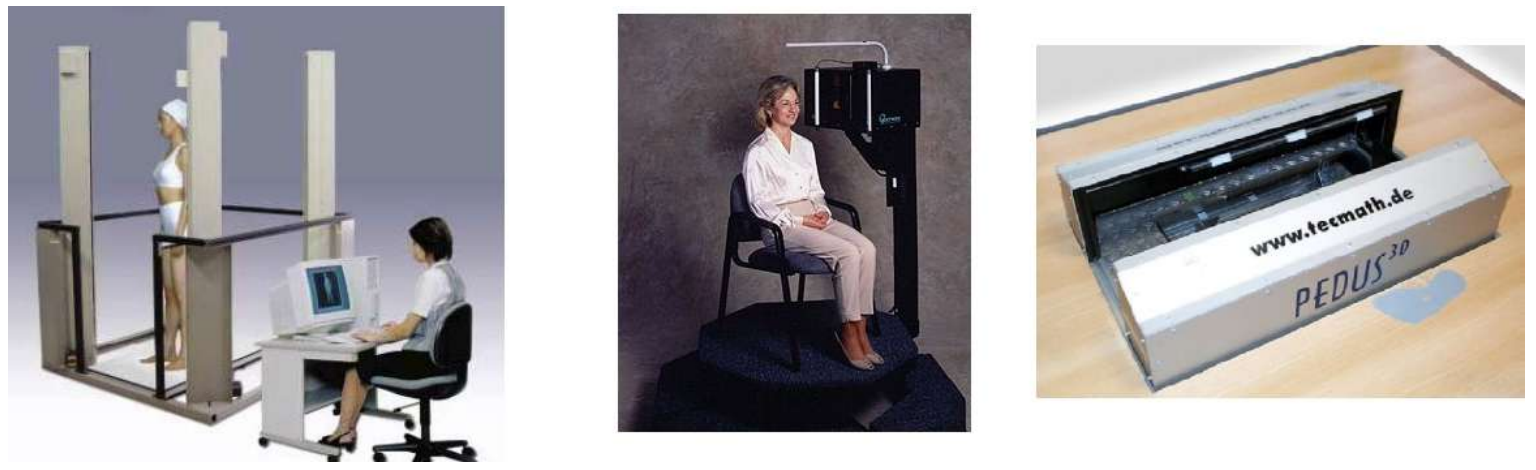

Figure 11: Laser scanning systems. Left: full body scanner BodyLine of Hamamatsu (Japan). Center: head scanner Head \& Face 3030 $3 D$ Color Scanner of Cyberware (USA). Right: foot scanner Pedus of Vitronic (Germany).

\subsection{Projection of light patterns}

The second technology used extensively for human body measurement is based on the projection of light patterns. It comes closer to the solution of the problems described above. Instead of moving the scanner unit, a light pattern (usually in form of stripes) is projected onto the human body (Figure 12 left). A light sensor (e.g. a digital camera) acquires the scene. The scanning device is composed usually of a pattern projector and a light sensor (Figure 12 center). More complex systems use two or three light sensors. The measurement process is similar to the method of laser scanning: stripes on the surface are measured singularly by using triangulation. The difference is that this happens in a single step and that the entire surface can be digitized by a single acquisition. For the increment of the resolution, the projected stripes are shifted and multiple scenes are acquired. Everything happens in short time period (mostly under one second), so that human bodies can be digitized without problems: the uncontrolled movements of the person are not a problem. However, the field of measurement of such scanning devices is limited, e.g. Capturor of InSpeck (Canada) (Figure 12 center) can measure surfaces with maximal size of half part of the human body (e.g. upper torso). To measure large parts of the human body (e.g. entire head, full body) multiple scanning devices are required. Figure 12 shows on the right an example of a full body scanning system composed of six scanning units. This procedure has the disadvantage, that multiple units cannot be used simultaneously since they interfere with each other's light patterns projections. Practically, this means, that multiple equipments have to be used serially. This implies again an extension of the acquisition time. More advantageous, in comparison to laser scanning, are instead the today's costs of the technology.
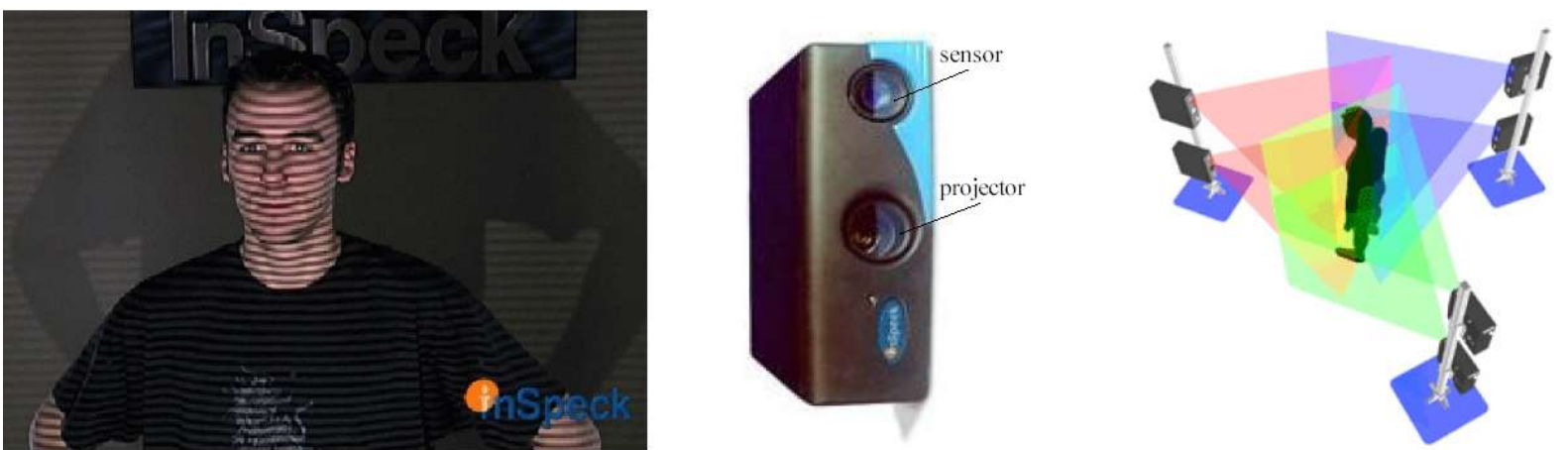

Figure 12: Left: projection of light pattern as stripes. Center: scanning device Capturor of InSpeck (Canada). Right: six Capturors compose a full body scanning system.

\subsection{Image processing and modeling}

The third technology utilizes image processing and modeling techniques for the digitization of the human body. In this case, $3 \mathrm{D}$ measurements are not performed, but 3D information is generated and extracted from $2 \mathrm{D}$. Two examples are described to explain this technique: the 2D full body scanner Contour of Human-Solutions (Germany) (Figure 13 left) and the face modeler FaceGen of Singular Inversions (USA) (Figure 13 right). By the first example, three images of a person are acquired (two from the front and one from the side). By using the symmetry of the human body, the most important sizes of body are computed with sufficient accuracy from the silhouettes of the body. The extracted body sizes are used, in this specific example, for the preparation of mass customized dresses. 

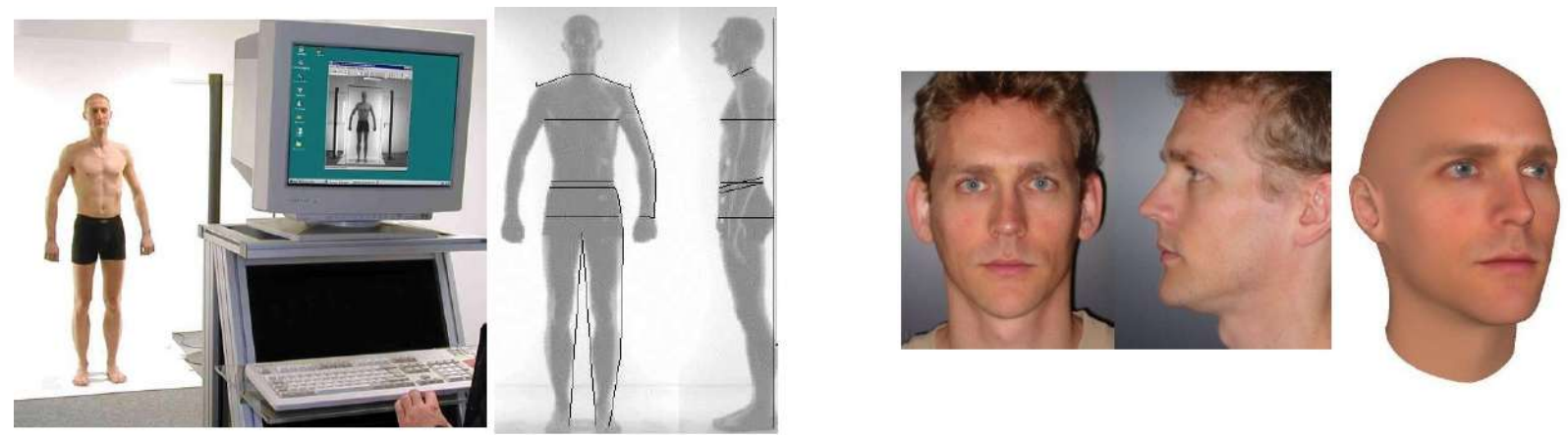

Figure 13: Left: 2D Full body scanner Contour of Human-Solutions (Germany), scanning equipment and silhouettes images used for the measurement. Right: face modeler Facegen of Singular Inversions (USA), used images for the generation of the virtual head and generated 3D face model with texture.

The second example shows the possibility to generate extremely realistic face models by using only two images of the person (from the front and from the side). The 3D computer model is generated manually with the help of user-friendly software tools. In this case, a real measurement of the human face is not performed. However, the produced 3D computer models are extremely photorealistic and completely adequate for applications as, for example, animation and computer games. The big advantage of this combined technique (image processing and modeling) is its extremely lower price compared to real 3D measurement.

\subsection{Required and useful software}

For the completion of the overview on the actual state of the technology for the digitization of the human body, the required and helpful software has also to be described.

The raw data resulting by the scanning process can usually not be used in its original form. Mostly scanners are therefore provided with standard software for the visualization, for the treatment, for the exporting and eventually for the editing of the data. Figure 14, on the left, shows for example the result achieved by TriForm full body scanner of Wicks and Wilson (UK) and at the center the result of a head scanning system of InSpeck (Canada). It can be clearly noticed that raw data of the full body scanner has to be processed. In the case of the head scanner, the result shown as a colored 3D model of a female head has already been processed.
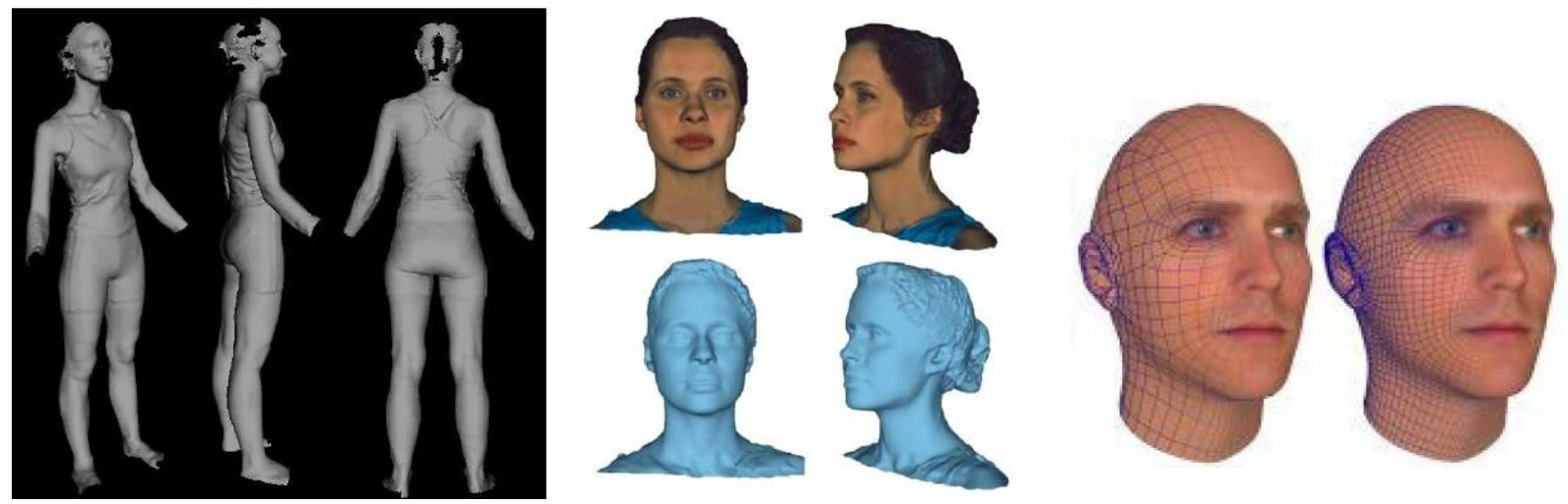

Figure 14: Left: result of TriForm full body scanner of Wicks and Wilson (UK). Center: head model (with and without texture) resulted by using InSpeck (Canada) head scanner system. Right: two different levels of detail of a 3D face model resulted by Facegen of Singular Inversions (USA).

Data compression plays an important role by the digitization of the human body. 3D scanning processes generate very large amount of data, e.g. the female head model in Figure 14 corresponds to about 27 Mbytes of data. Therefore, for an efficient and unproblematic storage, treatment and visualization of the data, adequate compression processes are required. These can be defined specifically to the different parts of the human body by considering the typical topology of their surface. We take the human face as example: every human face is round shaped, has a nose, a mouth, two eyes, etc. This basic information is used to reach strong compression factors without loosing the important features of the human face. Figure 14 on the right shows an example of a 3D face model at two different compression factors: the details of the eyes, nose, and mouth must be conserved, whereas the data resolution in areas with fewer details can be reduced. 


\section{APPLICATIONS AND EXPLOITATION OF HUMAN BODY DIGITIZATION}

\subsection{Introduction}

Various application fields exploit the digitization of the human body since many years. The different applications can be classified into two big groups, distinguishing them by their requirements:

- The first is more interested in the visual aspect of the results and regards mainly movie industry, animation, computer games, and virtual reality.

- The second, more interested in the quantitative aspect of the result, namely the measurement, includes ergonomics, anthropometrics, medical applications, and biometry.

The next paragraph will give some examples of the exploitation of human body $3 \mathrm{D}$ surface measurement techniques for various fields.

\subsection{Appeal / fashion / beauty}

Experts dealing with styling of persons are increasingly interested in commercial applications of human body digitization in their field. They wish to show their clients how they will look before they buy trendy dresses or before they choose for a beauty treatment. They also wish they could provide them with customized dresses at affordable costs. Figure 15 shows examples of existing applications in this field. On the left is shown a snapshot of a virtual fashion show with digitized human models. On the center is displayed a digital customer card with stored body sizes, that were determined by full body scanning process. On the right is shown a so called virtual-try-on solution, where different cloth items can be tried virtually on a 3D model of a person.
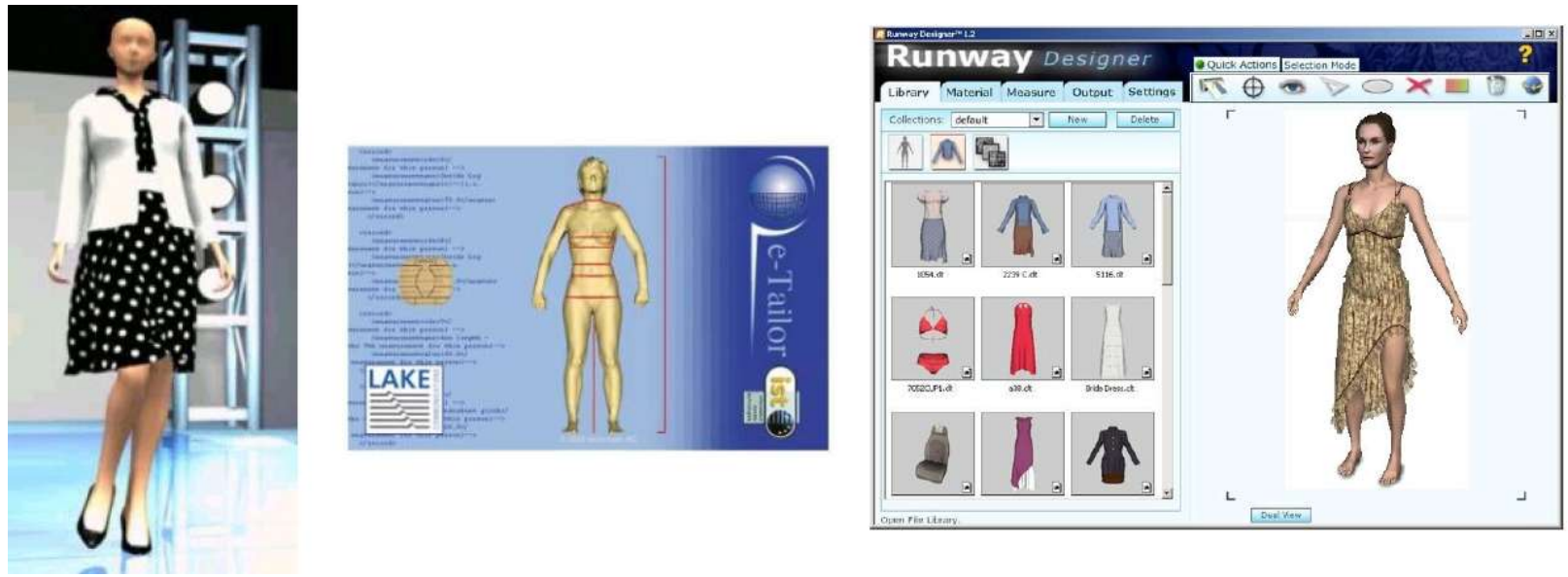

Figure 15: Examples of exploitation of human body digitization for styling applications. Left: virtual fashion show, from Digital Fashion (Japan). Center: digital customer card with stored body sizes, from e-Tailor project (EU). Right: 3D virtual-try-on solution, from Optitex (Israel).

Figure 16 and Figure 17 show other possible applications in beauty branches, namely hairdressing and accessories (e.g. glasses). In both examples, the appeal of the human face is changed virtually by adding digitally haircuts or glasses to the digitized 3D human face.
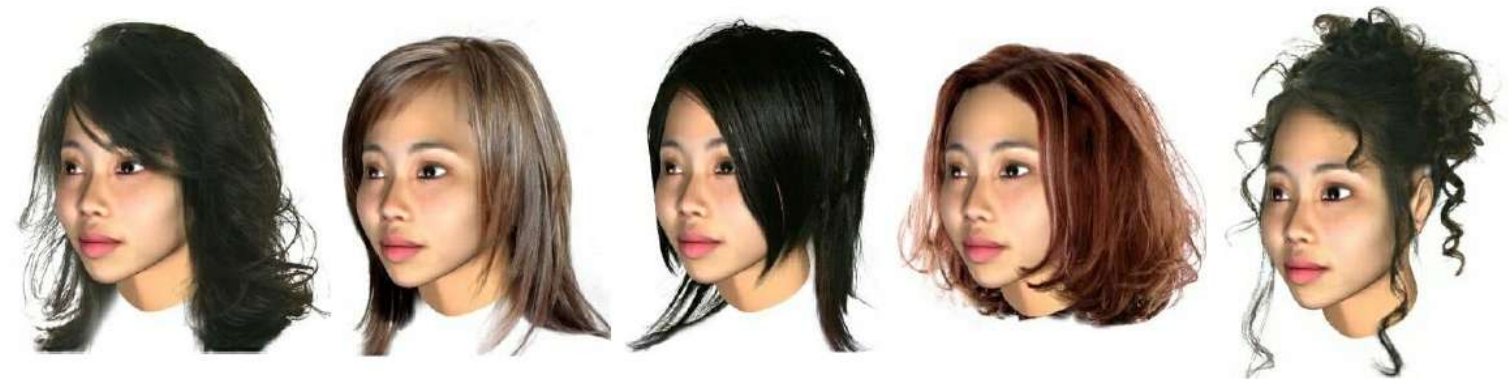

Figure 16: Example of applications in beauty: virtual added haircuts, from Stellure (USA). 

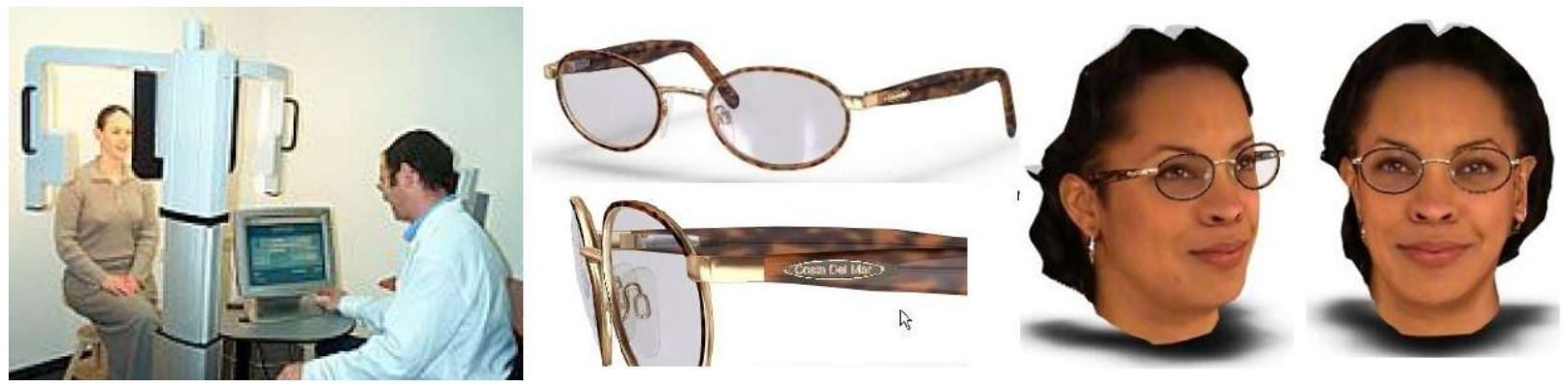

Figure 17: 3D iView (Israel) acquisition system (left); virtual 3D frame and lenses (center); 3D eyewear virtual-try-on (right).

In the case of virtual-try-on of eyewear, the benefits are manifolds for both customers and optical stores ${ }^{6}$. From the point of view of the client, the benefits are: the unique high tech buying experience, the quick and easy way to select frames, the time saved on frame pre-selection, the possibility to share the frame selection with family and friends, the ability to see himself with new frames without lenses, the fast virtual try-on of new collections, and the private selection by Internet without obligation or embarrassment. From the point of view of the optical store, the possible benefits are: the augmented attractiveness of the store due to a unique purchasing experience, the reduced number of rejected frames due to accurate measurement, the saving on stock by holding less frame inventory, the extended availability of large collection of frames due to the virtual inventory, and the optician time saved by obtaining pre-selection of frames by the client.

\subsection{Size surveying ${ }^{7}$}

Recently, human body size surveys have become a complete necessity. All institution and companies working on ergonomics are expecting information for practical immediate exploitation. It had been realized that anthropometric data could improve the quality of product design and usability, workstation and work place planning, and even laborer safety in certain environment. Establishing a national anthropometry data base for the nation's citizen is now undoubtfully a necessity.

National surveys have been initiated in the past in UK (SizeUK, 11'000 subjects scanned, 130 body measurement for each subject), in USA (SizeUSA), but recently also in Sweden and in France. The standard posture used in all surveys features the legs and arms slightly apart of the human body, elbows and hand joints slightly bent. This to allow an automatic determination of the important anthropometric measures of the human body (ISO7250). The most recent size surveys (Sweden and France) have added additional sitting and standing postures. New surveys lunched in China also added the 3D measurement of hands and feet.

The automatic recognition of anatomic landmarks as defined by ISO7250 works well for the standard posture, however some problems still remain for very fat people. On the other hand, research group are working for the development of algorithm for the automatic landmark recognition also for the sitting posture.
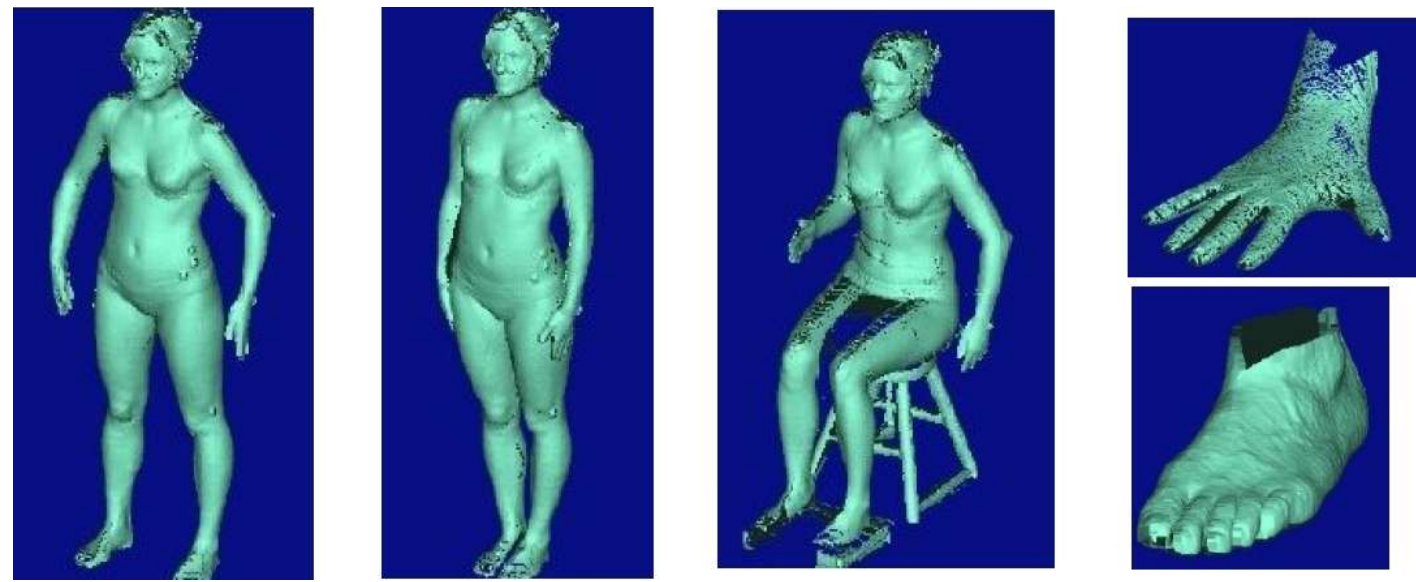

Figure 18: 3D data required by size surveying? : standard (legs, arms apart), standing and sitting postures, foot and hand. 


\subsection{Medicine}

The medical field represent an established group of users of human body 3D digitization techniques. Various applications exist already in orthodontics, prosthetics, orthopedics, plastic surgery, reconstructive medicine, forensic medicine, dentistry, ORL and cosmetics.

As commercial example for the medical field, Figure 19 shows the 3D measurement system for spine and posture analysis developed by a German company. The system is based on structured light projection. The very short scanning time of about $40 \mathrm{msec}$ allows the dynamic 3D measurement (4D) of the spine. The gained information is of great use for analysis and diagnosis.
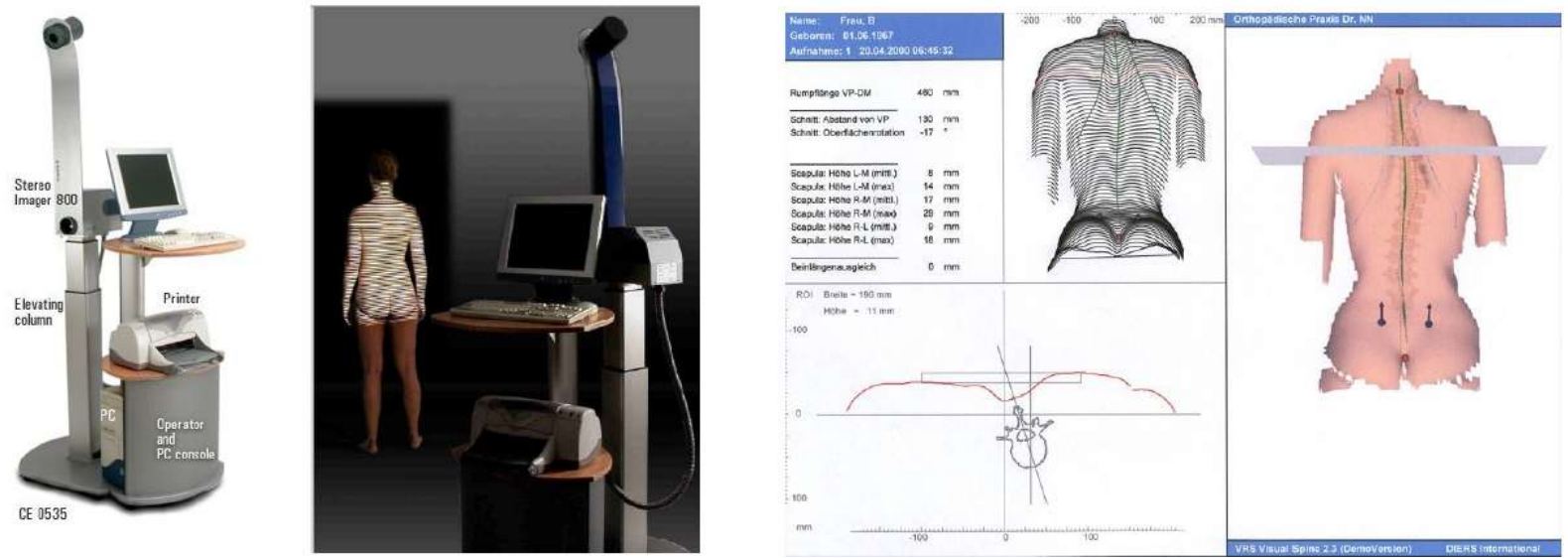

Figure 19: Human back measurement system Formetric 3D/4D (Germany). Complete system (left), system in use (center), application software (right).

\section{$5.5 \mathrm{Art}$}

Artists are becoming more interested in 3D human body digitization since the costs of this technology has decreased in the last years and became more accessible to a larger group of users.

3D replica of human faces or full body are offered in various media, as for example, point cloud burned in crystal cubes, or small plastic figurines printed in 3D. However, such replicas do not represent real artistic exploitation of 3D measurement techniques. A very interesting example of how artists can exploit the technology for their work is given by Helmick and Schechter's sculpture of year 2000, called Jurisprudent ${ }^{9}$ and installed at the Melvin Price Federal Courthouse in East St. Louis (IL, USA). Using traditional methods, the artists sculpted twelve life-size portraits of ordinary American citizensrepresenting twelve members of a jury. The heads were then laser-scanned, rapid-prototyped at small scale, cast in pewter in large quantities, finished with a hand-rubbed patina, and precisely affixed to hundreds of suspended cables. Collectively, the over 3,000 small sculptures coalesce into two monumental heads facing each other across a skylit courthouse atrium. Figure 20 shows the sculptures.
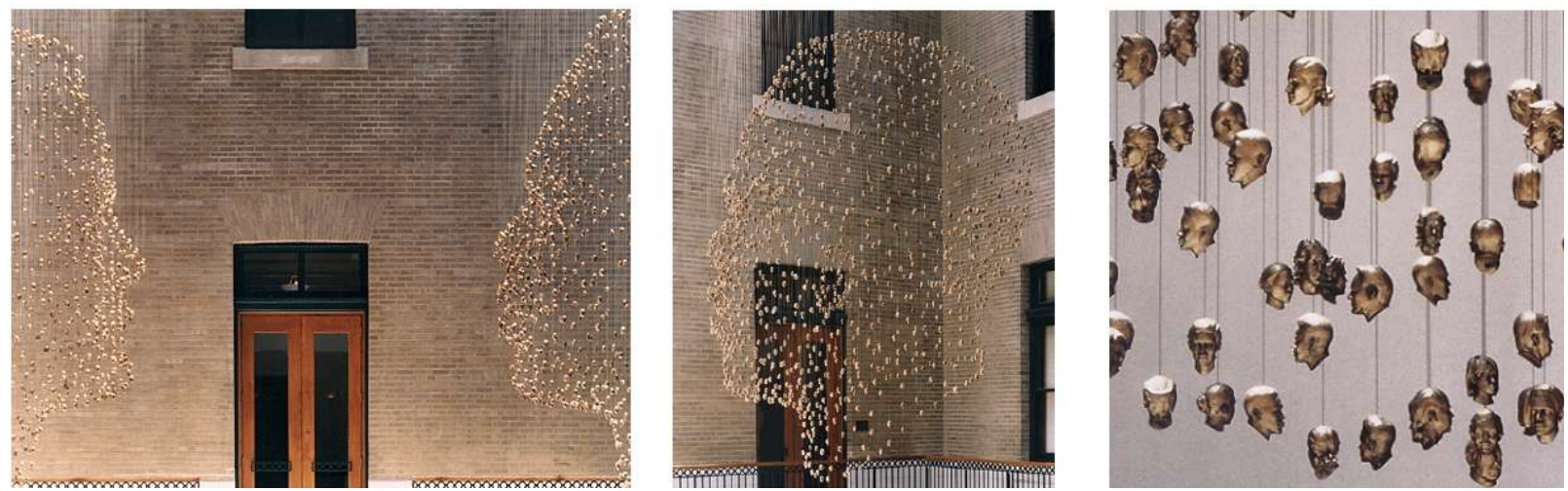

Figure 20: Artistic installation Jurisprudents, at the Melvin Prince Federal Courthouse (USA). Large view (left), the male face (center) and detail of the suspended small sculptures (right). 


\subsection{Animation}

The last example of application of 3D human body measurements come from the technological innovation exposition 2005 in Aichi, Japan. A world-first in entertainment was shown at the Mitsui-Toshiba Pavillon: Grand Odyssey, a unique computer animated film ${ }^{10}$. Spectators queuing outside what appeared to be an ordinary cinema, were invited to place their faces into a hole in the wall for a few seconds. High-resolution digital cameras performed a quick scan from several angles, after that everyone could take their seats. The animated film was beginning as normal, but the cast was made up of walking, talking digital replicas of people in the audience. Each spectator were getting a role (there were soldiers, doctors, scientists and politicians involved in the story) as a Toshiba supercomputer was processing the one-time-only film.

The system named Futurecast was initially developed by Waseda University Professor Shigeo Morishima, and completed by Mistui-Toshiba engineers. The entire process can be described as follows: (a) a simple face scanner capture images from the person from different directions; (b) a 3D computer model of the person's face is automatically generated from the scanning data; (c) out of the 3D face model a parametrized face mask is automatically extracted, the parametrization allows to index face features as eyes, eyebrows, nose, mouth, etc.; (d) the face mask can then be directly inserted and animated in the computer animated movie.
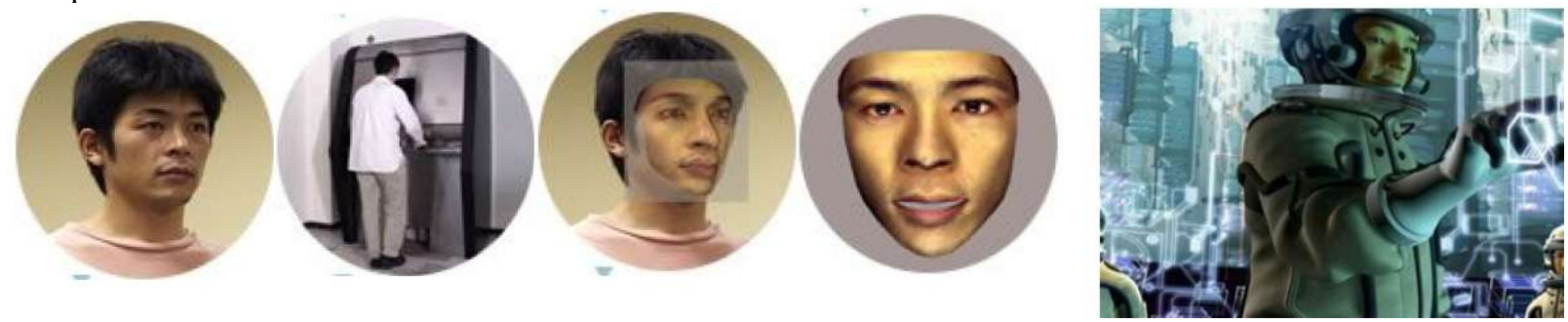

Figure 21: Futurecast system (Japan). Left: Spectator original face, face scanner, 3D face model and parametrized face mask. Right: person's face inserted and animated in real-time in the movie.

\section{CONCLUSION}

In this paper was presented an overview of the different 3D surface digitization technologies commercially available in the European market. Various examples of their use were shown for different application fields. Special interest was given to applications regarding the 3D surface measurement of the human body.

\section{REFERENCES}

1. Nub3D, Dimensional inspection of turbine blades, http://www.nub3d.com/english/aplicaciones03.htm (accessed 12/19/2005).

2. A. Bellmann, T. Suthau, S. Stoinski, A. Friedrich, O. Hellwich, H.-C. Gunga 3D-Modelling of dinosaurs, Proc. of the 7th conference on Optical 3-D Measurement Techniques, Vienna, Austria, October 3-5, 2005.

3. R. Heintz, H. Fahrner, G. Bono, R. Van Cauter, Inspection of plastic tool housings at the speed of light, http://www.metris.com/en/html/successtories/Atlas Copco Article ENG.pdf (accessed 12/19/2005).

4. 3Shape, D-200 Dental 3D scanner, http://www.3shape.com (accessed 12/19/2005).

5. N. D'Apuzzo, Digitization of the human body in the present-day economy, in: Beraldin, J.-A., El-Hakim, S.F., Gruen, A., W alton, J.S. (Eds.), Videometrics VIII, Proc. of SPIE-IS\&T Electronic Imaging, SPIE Vol. 5665, San Jose (CA), USA, 2004, pp. 252-259.

6. Visionix, Visionix 3D iView, The world's first commercial 3D virtual-try-on system for eyewear, Human Body Measurement Newsletter, Vol.1, Nr.2, September 2005, http://www.homometrica.ch/en/indexnews.html (accessed 12/19/2005).

7. J.-M. Surville, Advances in 3D acquisition, Proc. of 3D modelling 2005, September 27-28, 2005, Paris, France.

8. Diers, Optical stato-dyamical spine analysis and posture measurement system: formetric 3D/4D, http://www.diers.de/english/measurement/formetric/formetric.htm (accessed 12/19/2005).

9. Helmick and Schechter Sculpture, Jurisprudents, http://www.handsart.net/jurisprudents.html (accessed 12/19/2005).

10. N. D'Apuzzo, Grand Odyssey, the film where spectators star in, Human Body Measurement Newsletter, Vol.1, Nr.1, April 2005, http://www.homometrica.ch/en/indexnews.html (accessed 12/19/2005). 